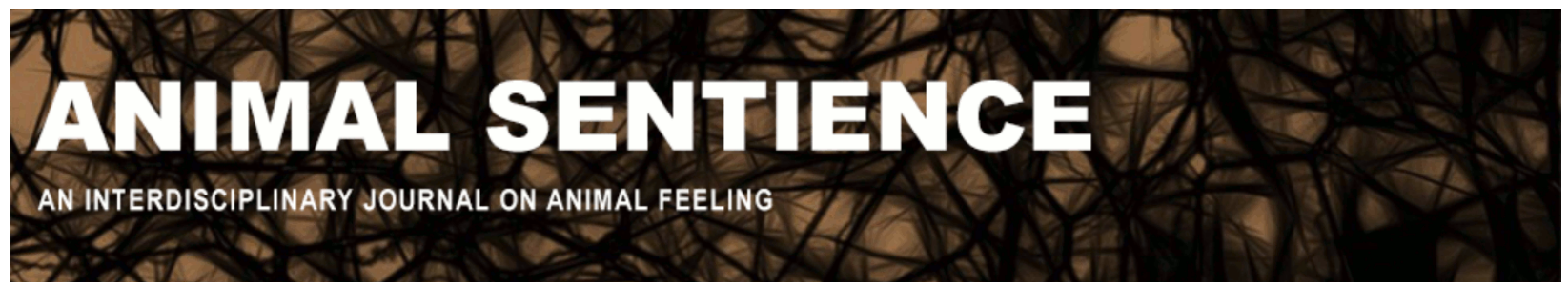

Key, Brian (2016) Burden of proof lies with proposer of celestial teapot hypothesis. Animal Sentience 3(44)

DOI: $10.51291 / 2377-7478.1094$

Date of submission: 2016-02-21

Date of acceptance: 2016-02-26 (c) 


\title{
Burden of proof lies with proposer of celestial teapot hypothesis
}

Response III to Commentary on Key on Fish Pain

\author{
Brian Key \\ School of Biomedical Sciences \\ The University of Queensland
}

\begin{abstract}
Bertrand Russell famously imagined the existence of a celestial teapot to highlight that the burden of proof of a hypothesis lay with its proposer and it was not the responsibility of others to refute it. Those who propose that fish feel pain must bear the burden of proof for their hypothesis. There are several common arguments adopted by those defending the position that fish feel pain. For instance, proponents envisage that pain is so important for human survival that they can't imagine fish could exist without it. Out of this argument from incredulity emerges the idea that pain must have evolved early in animal evolution ergo fish feel pain. Fish pain proponents often accept that fish might feel pain because it seems highly probable that fish do. Similarly it is reasoned that if fish seem to display multiple human-like characteristics (such as complex behaviours, learning and tool use), then they must be intelligent and hence make it more likely than not that they feel pain. It has been argued that those who accept the null hypothesis that fish do not feel pain are merely cherry picking the literature to refute fish pain. These claims are levied without understanding that the strength of inferences is heavily influenced by the robustness of the data and the nature of the results. The fallback position that fish pain proponents adopt when they accept that the fish forebrain does not generate pain is to suggest that fish pain arises from somewhere else in the brain using different circuitry. In the case of the present commentaries the brainstem is recognised as the seat of pain in fish. Although it is not the responsibility of the non-proposer of this "celestial teapot" to refute it, I provide evidence that makes continued adoption of this stance untenable.
\end{abstract}

Brian Key brian.key@uq.edu.au is Head of the Brain Growth and Regeneration Lab at University of Queensland. He is dedicated to understanding the principles of stem cell biology, differentiation, axon guidance, plasticity, regeneration and development of the brain.

http://www.uq.edu.au/sbms/staff/brian-key

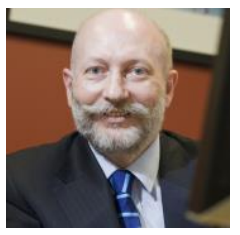

Bowers proposes that the question of whether fish feel pain can be answered by a simple experiment involving an unconditioned stimulus devaluation paradigm. The most serious flaw in this strategy (as pointed out in the editorial note preceding it) lies in the fact that it is built on the false premise that an internal representation of an unconditional stimulus (e.g., electric shock) is necessarily conscious.

Brown falsely argues by "appeal to popularity" that fish most likely feel pain. Such an approach (also colloquially known as the "bandwagon effect") is fallacious. Brown further falls into the trap of the logical fallacy of the "appeal to consequences" by attempting to suggest that accepting fish do not feel pain means that these animals are consequently not capable of learning. 
Cooke indicates that the "commentaries are rife with bias and represent a potentially dangerous form of advocacy." He recommends that a better way forward is to engage a learned academic society to undertake an unbiased systematic review of the literature. However, such an approach is easily tainted by the "appeal to popularity" argument. It is not unusual for science at the leading edge to be engulfed by controversy and plagued by deep divisions of opinion. History has shown that with time these issues resolve and the field moves forward.

Damasio \& Damasio argue for fish feeling pain based on four principal reasons. First, they contend that pain occurs in the brainstem and not the cortex. However, as already indicated in the target article, one should not uphold motor outputs from the brainstem as evidence of pain. Second, Damasio \& Damasio fail to understand why cortical stimulation should establish an exclusive role for the cortex in pain. Without reiterating the evidence already presented in the target article, I will highlight some insightful experiments conducted in rats that demonstrate the cortical, rather than brainstem, origin of pain (see references in Key, 2015a).

Rats injected with the chemical irritant formalin into their feet display paw shaking, licking and grooming, and act to limit contact of the injected foot with the ground. When these animals are decerebrated they continue to exhibit these behaviours. A non-critical interpretation of these results is that the animals feel pain at the level of the brainstem and not the cortex (which is what Damasio \& Damasio proclaim). However, systemic injection of morphine fails to reduce the motor responses in these decerebrated animals. If pain did arise from the brainstem, then one would predict that morphine would reduce the sensation and hence the protective behaviours. However, since the behaviours were not affected, it is unlikely that the brainstem was generating the sensation of pain. Systemic morphine was active and capable of inhibiting the behaviours, but only in sham-operated controls in which connections between the brainstem and forebrain were spared. Thus, the forebrain seems essential for pain-like behaviours. The role of specific cortical regions was subsequently confirmed when it was shown that protective behaviours were attenuated by localized injections of morphine into the cortex. The idea that pain arises in the brainstem is clearly untenable.

Damasio \& Damasio's third conjecture is merely to hypothesise that "some grade of experience could emerge from complex subcortical nuclei." The burden of proof for this hypothesis lies with the authors, particularly given the considerable evidence contradicting it (Key, 2015a; Key 2016a). Damasio \& Damasio agree with the target article that pain may persist when a cortical region is damaged because pain involves a network of cortical regions. Nonetheless, they disregard that supra-brainstem regions generate pain because there are supposedly few reports in humans of loss of pain with thalamic damage. However, there is a long history of clinical evidence beginning with seminal observations by Head and Holmes (1911) - see Craig, 1996, and Price et al., 2006, for more detailed discussion on this matter - that supra-brainstem regions (i.e., thalamus and cortex) produce pain in humans. (I further discuss this point below.)

In their final conclusion, Damasio \& Damasio indicate that the insular cortex does make an important contribution to pain but only by allowing that pain to be communicated. Damasio \& Damasio appear to be defending the position that the cortex merely communicates the presence of pain while the feeling itself arises in the brainstem. This line of reasoning can be likened to the suggestion that the conscious feeling of pain is generated in the spinal cord of paraplegics but it is not verbally reported as pain because the transmission between the cord and cortex is severed. If 
this was the case, then direct cortical stimulation should not elicit pain (which it can; Key 2016a) because communication with the site of pain generation would be lost. It should be noted that discrete lesions of the thalamus in humans (Hitchcock and Teixeira, 1981; Laitinen, 1988; Hariz and Bergenheim, 1995; Abosch and Lozano, 2004; Weiss et al., 2005) can produce analgesia, indicating that supra-brainstem regions are responsible for the feeling of pain. The failure of Damasio \& Damasio to recognize the vast surgical literature on the use of thalamic lesions to relieve pain reveals a level of bias. Interestingly, in their final sentence Damasio \& Damasio once again advocate subforebrain mechanisms by suggesting that feelings may actually arise in the peripheral nervous system and the enteric nervous system (which is actually part of the peripheral nervous system). Again the burden of proof lies with Damasio \& Damasio to substantiate such vagarious claims.

Devor, like Damasio \& Damasio, is unable to dissociate motor responses generated by the brainstem from the conscious experience of pain that arises in the cortex. However, the results from the lesion and morphine experiments on the formalin-model of pain in rats provide a clear demonstration of this distinction. Devor attempts to paint a picture that suggests that our understanding of human pain is too rudimentary to allow inferences to be drawn with regard to fish. To assist the reader, I will succinctly summarise some major findings arising from over 100 years of clinical and experimental work that further shines light onto the cortical origins of pain.

The spinothalamic tract is a major ascending axon tract that carries noxious information from the dorsal horns of the spinal cord to thalamic nuclei. This tract passes through the brainstem en route to the thalamus. Thalamic fibres then subsequently course into the cortex. (See Hong et al., 2010, for reconstructions of this pathway in live human brains.) If the spinothalamic tract is discretely severed anywhere along its length (or alternatively, if its terminal nuclei in the thamalus are lesioned), it causes analgesia in humans and other mammals. A recent clinical case report clearly demonstrates that lesioning the spinothalamic tract provides immediate relief of pain (Ivanishvili et al., 2016). If pain was arising from the brainstem, then selective severing of the spinothalamic tract above the brainstem should not affect pain. In these experiments the brainstem remains intact as do connections between the brainstem and the thalamus and cortex. This refutes the claim by Damasio \& Damasio that the cortex is merely a conduit for the communication of pain. One cannot then suggest that noxious information passes via the spinothalamic tract to the thalamus and then to the brainstem (and the loss of the spinothalamic tract would hence prevent noxious stimuli from reaching the brainstem). It should be pointed out that there is a separate spinoreticular pathway that also carries noxious information from the spinal cord to the brainstem (Willis, 1985). Thus, the results of severing the spinothalamic tract provide an additional line of evidence that refutes the idea that the brainstem is the generator of pain.

Devor claims that because the cerebellum can become active during pain one would expect the cerebellum to be directly involved in pain. However, he then dismisses this because electrical stimulation of the cerebellum does not produce pain. It is well known that the cerebellum is activated by noxious stimulation via a spino-bulbarcerebellar pathway and that this allows regulation of motor behaviour in response to noxious stimuli (Huma et al., 2015). Devor has inadvertently highlighted the fact that direct electrical stimulation is an additional test for the involvement of the cortex in pain. 
Devor proposes that the human cortex is a modifier of pain rather than a generator. This hypothesis predicts that direct stimulation of the cortex will not produce pain. He cites numerous examples where stimulation of the cortex fails to cause pain. These are negative results that lead to weak inferences because it can be difficult to ascertain whether the lack of positive results (i.e., pain) was due to problems with the experimental procedure (e.g., difficulty in stimulating the pain circuitry). However, once a positive result is obtained the hypothesis is immediately falsified (this is a strong inference). One is reminded of the analogy that "all swans are white" but once a black swan is found then the hypothesis is not supported.

Given that pain has repeatedly been shown to be experienced following direct cortical stimulation (the reader is encouraged to view the video of a patient's response to direct cortical stimulation, which is available as supplementary online material in Mazzola et al., 2012), we are justified in strongly inferring that the cortex is not merely a modifier. One can then propose and test the hypothesis that the cortex is the generator of pain. A prediction of this hypothesis is that the ablation of appropriate cortical regions should lead to a perturbed experience of pain. Positive results have been reported for this experiment and hence we have further support for the inference that the cortex is the generator of pain. By ignoring the positive responses and only accepting the negative outcomes Devor is trapped by cognitive bias. This line of thinking causes him to neglect the rodent work (see Key, 2016a) that further indicates that the cortex is the generator of pain.

Diggles concurs with Derbyshire in calling attention to the mischievous use of the term "pain" to describe the reaction of fish to noxious stimuli. He further highlights issues of fish welfare that are not dealt with by the notion of fish pain.

Edelman points out that "structural and functional similarities to the vertebrate hippocampus have been noted in the neural circuitry subserving memory in certain insects." It is not clear what Edelman is suggesting here given that this statement (if true) is supportive of my line of reasoning. He extends his discussion by raising the structural similarities of the eye in octopi and in vertebrates. I also raised similarities in the structure of olfactory glomeruli as well as pattern generators across species to highlight how structure determines function (Key, 2016a). The conclusion by Edelman is then a contradictory statement that "pain perception in bony fish might have been initiated via circuitry distinct from the mammalian pathways." The burden of proof lies with Edelman and it must be stronger and more convincing than "might have been initiated" to reject the null hypothesis that fish do not feel pain. (This harks back to the celestial teapot hypothesis.)

Merker reiterates his idea that the tectal-periaqueductal complex (part of the brainstem) "might house the circuitry through which nocifensive behaviour is attended by pain." As I said above, the burden of proof for a hypothesis calls for more evidence than that the brainstem "might house" the circuitry. I have already discussed some additional literature that further demonstrates that pain is not generated in the brainstem. However, it is noteworthy that Merker has restricted his argument to the notion that the brainstem is controlling the motor behaviours rather than the pain per se. Merker queries whether the neuroanatomy of the bird pallium is consistent with my four - not 21 as imagined by Merker - principal neuroanatomical criteria. He questions lamination because it seems to be missing in birds. To save space and so as not 
to seem to be ignoring these comments, I refer Merker to the original papers on the bird pallium (as discussed in Key, 2015a) in order that he better understands this matter.

Merker claims that I made "an astonishing misreading of the cited study" when I suggested that Yager et al. (1977) had shown that tectal ablation does not affect escape responses to touch. On page 271 of the Yager et al. paper, they report that tectumless fish exhibited "an exaggerated startle" (i.e., avoidance or escape) "response when they were touched with the net." So not only do tectumless fish continue to respond to touch as I stated, but they also actually exhibit a heightened response. Merker inappropriately claims, using the fact that "visually conditioned ... breathing responses to electric shock are abolished" (Yager et al., 1977) in tectumless fish, that the tectum is therefore involved in feeling the electric shock. However, these results merely demonstrate that the tectum was necessary for perception of the conditioning light stimulus. Merker's misjudgement in this case is due to his not fully comprehending the technical approach. Yager et al. did not assess the role of reinforcing electric shock on visual performance after tectal ablation. A follow-up study by Davis and Klinger (1987) (that should have been cited in Key, 2016a) addresses this matter. Using the same experimental method as Yager et al., Davis and Klinger showed that tectumless fish still responded to electric shock. They revealed that an electric shock was needed to reinforce spatial discrimination by both sham-operated control fish and tectumless fish. Thus, tectumless fish can still respond to electric shock, meaning that the tectum is not directly involved in nociception (and certainly not pain). Merker now needs to "budge" and provide some substantial evidence to support his hypothesis that the tectal-periaqueductal complex "might house" the necessary circuitry for pain in fish.

Merker attacks my citing Lovejoy and Krauzlis (2010) regarding lack of evidence that the tectum contributes to conscious awareness. Merker proclaims that there is not one word about conscious awareness in this paper. (That turns out to be precisely the point I am making.) The primate superior colliculus is involved not only in the motor control of eye saccades, but also in the selection of the appropriate target in the visual field where the saccades will direct attention. Lovejoy and Krauzlis show that attention can be directed toward an object in the visual field without an eye saccade (i.e., gaze) to that object (so-called covert attention). While previous studies have shown that the cortex is involved in covert attention, there has been no evidence that the superior colliculus is involved. Lovejoy and Krauzlis provide that evidence. However, there is still no evidence, as yet, to indicate that the superior colliculus is directly responsible for conscious awareness of the target in the absence of the cortex. It should be noted that the Lovejoy and Krauzlis citation was introduced as part of a response to a previous commentary from Merker and was not discussed in the target article (Key, 2016a). The burden of proof for Merker's hypothesis still remains and that proof needs to be considerably more convincing than attempts to undermine the appropriateness of two cited references.

Safina provides a series of anecdotes, just-so stories, engages in anthropomorphism and mixes in a series of fallacious arguments (such as argument from incredulity) to superficially defend fish pain. I have discussed most of these issues before (Key, 2015b; Key 2016a; Key 2016b; Key, 2016c) but Safina does raise a novel twist by questioning why some fishes have defensive spines if their predators don't feel pain. This question emerges from the anthropomorphic tendency to think that fish can only avoid an object if they feel it. (I discussed avoidance learning in the absence of conscious awareness in Key 2016a.) Safina then questions why a stingray's spine would also evolve venom, if 
not to produce pain. I remind the reader that noxious stimuli can work via direct local lesions (such as a stingray barb) as well as by venom that spreads more widely through tissue extracellular fluid and the lymph system (and thereby activates a larger tissue mass). Venom and barbs are two different noxious stimuli for activating the nervous system.

Walters refreshingly applies the structure-function approach to squid. However, there is a danger in using this approach across distant lineages that possess anatomically incongruous nervous systems. While I don't have the space here to argue for, or against, every animal model, I do ask that the argument go beyond black box analogies. A reasonable attempt should, at least, first identify where in the squid nervous system pain is supposedly generated and second, describe how that circuitry is consistent with the neural processing underlying pain. I accept that the size of the brain is not important but that rather the neural tissue should possess the necessary neuroanatomy to be capable of being aware of its own neural activity and to experience it as pain (or by any other name that it is camouflaged as, e.g., "raw experience").

Walters argues that squid have their own pain that is a "raw experience" and proposes that, as such, it does not require the sophisticated circuitry of mammals. However, the fundamental notion of pain, as opposed to nociception, is the awareness of the stimulation as something "that it feels like." To put this in colloquial terms, "feelings must be felt." It is this "what it feels like" that requires a specific type of neurocytoarchitecture and interconnectivity. Walters claims that because many animal nervous systems contain networks and circuits that could be associated with signal amplification and global integration, then they "may experience forms of conscious pain." Interestingly, Walters did not sufficiently elaborate on the nature and whereabouts of these circuits. In an attempt to adjust the structure-function argument from vertebrates to invertebrates Walters has drastically oversimplified the approach. He has inappropriately reduced the necessary fundamental properties to the simple requirement for coordinated widespread activity of neurons.

Walters calls for the use of conditioned place preference paradigms to demonstrate pain in fish and invertebrates. However, as previously discussed (Key, 2016c) this experimental approach is not insightful. Walters, like many other commentators (Key, 2016b), is guilty of using just-so stories. He imagines squid to have awareness of arm injury and as a result of this ability they are proposed to be able to realize that they are more vulnerable to predators. As a consequence, they choose to maintain normal activities to bring less attention to themselves.

The fact that Walters talks of "motivational states triggered by noxious stimuli" leads him to accept, inadvertently and prematurely, that the behaviours involve conscious awareness (that is: state $\mathrm{A}$ is motivational; motivation requires conscious awareness of goals; noxious stimuli cause state A; therefore, animal in state A is conscious). An entirely different interpretation would result if the state were instead referred to as a "motor output." Notably, other invertebrate researchers are more discerning about interpreting nocifensive behaviours in cephalopods and warn that they do not demonstrate pain-like states (Alupay et al., 2014).

Making sense of the senses. Some commentators seem concerned that accepting that fish do not feel pain has slippery-slope consequences. If fish do not feel pain, then perhaps they do not experience other sensory stimuli either. While this logic is understandable, what seems to be more troublesome for them is that fish may 
consequently be labelled as "zombie-like" or robotic. Putting such slippery-slope arguments aside, is it possible that fish do not consciously experience the colour red [i.e., that they are not feeling anything at all when they are receiving or responding to optical input]? It is not my intent to fully address this question here, as it will no doubt lead us too far from the primary topic of fish pain. However, given the interest in the general question I will briefly describe several key experimental results that should challenge readers to think more deeply about the issue.

1. It is uncontroversial that humans require the primary visual cortex (V1) to be aware of objects in their visual field. However, about 100 years ago it was realised that some World War I soldiers that were blinded by shrapnel injury to their occipital lobes (where V1 is located) were still able to somehow perceive the presence of moving objects (Riddoch, 1917). Subsequently, some individuals have been reported to navigate corridors littered with obstacles despite being blind. Some patients can also guess the presence of moving objects without being consciously aware of their presence. This nonconscious visual ability is referred to as blindsight (Weiskrantz et al., 1974). It seems that a neural pathway from the thalamus to middle temporal lobe (that bypasses V1; Barbeben et al., 2014), that is not accessible to conscious awareness, is responsible for this residual visual ability. What this means is that visual behaviour in humans is not totally dependent on conscious experience. The association between conscious experience and behaviour, which many people seem to intuitively think is mandatory, is not necessarily essential. Blind individuals can exhibit behavioural responses to objects in their visual field without consciously seeing (experiencing) them. This begs the question as to whether nonhuman animals could possibly do the same.

2. When the primary visual cortex has been injured in monkeys and rodents, these animals can also demonstrate nonconscious vision (Weiskrantz, 1996; Carey et al., 1990).

3. Toads can duck, turn away and avoid objects when their forebrains have been completely removed (Ewert, 1970).

4. Now what about fish? Fish swim and feed normally following removal of their entire forebrain. They can catch pellets of fish food without a forebrain. They can swim and flee to avoid capture with a fishnet without a forebrain. They can also respond to light signals during avoidance learning paradigms without a forebrain. Clearly fish demonstrate some remarkable nonconscious visual abilities. These results strongly support the idea that it is not necessary for fish to consciously experience sensory stimuli (whether noxious electric shocks or coloured objects) in order to respond behaviourally to them. One may retort by saying that the seat of all conscious experience lies below the level of the forebrain. But I will leave this up to the reader to think through carefully, especially now that we better understand the feedforward reflex-like pathways in the brainstem that drive behaviours such as prey recognition in zebrafish (e.g., Bianco and Engert, 2015).

As already noted, I do not describe the above results in detail but merely present them here to provoke and challenge researchers to think critically about how they intuitively respond to anecdotal observations of animal behaviour.

Some commentators raise questions about the evolutionary significance of pain in humans and other mammals. It seems inconceivable to some people that fish could survive for more than 400 million years without conscious experience of noxious stimuli. This question is a derivative of a much larger question about the purpose of consciousness itself. "Why" questions are always controversial; books and journals are full of discussions on why phenomenal consciousness [i.e., sentience] exists. One thing is 
certain: understanding the evolutionary (as well as developmental) origins of conscious awareness of noxious stimuli will bring us closer to deciphering its neural basis and perhaps also to appreciating its functional significance.

\section{References}

Abosch, A. and Lozano, A.M. (2004) Stereotactic ablative procedures for pain relief. Semin. Neurosurg. 15:195-202.

Alupay, J.S., Hadjisolomou, S.P. and Crook R.J. (2014) Arm injury produces long-term behavioral and neural hypersensitivity in octopus. Neurosci. Lett. 558:137-142.

Barleben, M., Stoppel, C.M., Kaufmann, J., Merkel, C., Wecke, T., Goertler, M., Heinze, H-J., Hopf, J-M. and Schoenfeld, M.A. (2014) Neural correlates of visual motion processing without awareness in patients with striate cortex and pulvinar lesions. Hum. Brain Map. 36:1585-1594.

Bianco, I.H. and Engert, F. (2015) Visumotor transformnation underlying hunting behavior in zebrafish. Curr. Biol. 25:1-16.

Bowers, R.I. (2016) Devaluation as a strategy to address behaviourally whether fish feel pain. Animal Sentience 2016.073.

Brown, C. (2016) Fish pain: An inconvenient truth. Animal Sentience 2016.058.

Carey, D.P., Goodale, M.A. and Sprowl, E.G. (1990) Blindsight in rodents: The use of a 'high-level' distance cue in gerbils with lesions of the primary visual cortex. Behav. Brain Res. 38:283-289.

Cooke, S.J. (2016) Spinning our wheels and deepening the divide: Call for an evidencebased approach to the fish pain debate. Animal Sentience 2016.072.

Craig, A.B. (1996) Pain, temperature, and the sense of the body. In Somesthesis and the Neurobiology of the Somatosensory Cortex (pp. 27-39). Birkhäuser Basel.

Damasio, A. and Damasio, H. (2016) Pain and other feelings in animals. Animal Sentience 2016.059.

Davis, R.E. and Klinger, P.D. (1987) Spatial discrimination in goldfish following bilateral tectal ablation. Behav. Brain Res. 25:255-260.

Derbyshire, S.W.G. (2016) Fish lack the brains and psychology for pain. Animal Sentience 2016.025.

Devor, M. (2016) Where is the pain in the brain? Animal Sentience 2016.060.

Diggles, B.K. (2016) Fish pain: Would it change current best practice in the real world. Animal Sentience 2016.061. 
Edelman, D.B. (2016) Leaving the door open for fish pain: Evolutionary convergence and the utility of 'just-so stories'. Animal Sentience 2016.062.

Ewert, J.-P. (1970) Neural mechanisms of prey-ctaching and avoidance behaviour in the toad (Bufo bufo L.). Brain Behav. Evol. 3:36-56.

Hariz, M.I. and Bergenheim, A.T. (1995) Thalamic stereotaxis for chronic pain: ablative lesion or stimulation. Stereotactic. Funct. Neurosurg. 64:47-55.

Head, H. and Holmes, G. (1911) Sensory disturbances from cerebral lesions. Brain 34:102-154.

Hitchcock, E.R. and Teixeira, M.J. (1981) A comparison of results from center-median and basal thalamotomies for pain. Surg. Neurol. 341-351.

Hong, J.H., Son, S.M. and Jang, S.H. (2010) Identification of spinothalamic tract and its related thalamocortical finers in human brain. Neurosci. Lett. 468:102-105.

Huma, Z., Ireland, K. and Maxwell, D.J. (2015) The spino-bulbar-cerebellar pathway: activation of neurons projecting to the lateral reticular nucleus in the rat response to noxious mechanical stimuli. Neurosci. Lett. 591:197-201.

Ivanishvili, Z., Pujara, S., Honey, C.M., Chang, S. and Honey, C.R. (2016) Stereotactic mesencephalotomy for palliative care pain control: A case report, literature review and plea to rediscover this operation. Brit. J. Neurosurg. 1-4.

Key, B. (2015a) Fish do not feel pain and its implications for understanding phenomenal consciousness. Biol. Philos. 30:149-165

Key, B. (2015b) Why fish (likely) don't feel pain. Scientia Salon Feb $5^{\text {th }}$ https://scientiasalon.wordpress.com

Key, B. (2016a) Why fish do not feel pain. Animal Sentience 2016.003.

Key, B. (2016b) Going beyond just-so stories. Animal Sentience 2016.022.

Key, B. (2016c) Falsifying the null hypothesis that "fish do not feel pain". Animal Sentience 2016.039.

Laitinen, L.V. (1988) Mesencephalotomy and thalamotomy for chronic pain. Modern Stereotactic Neurosurgery. Springer U.S.A. pp. 269-277.

Lovejoy, L.P. and Krauzlis, R.J. (2010) Inactivation of primate superior colliculus impairs covert selection of signals for perceptual judgements. Nature Neurosci. 13:261-267.

Mazzola, L., Isnard, J., Peyron, R. and Mauguiére, F. (2012) Stimulation of the human cortex and the experience of pain: Wilder Penfield's observations revisited. Brain 135:631-640. 
Merker, B. (2016) How not to move the line drawn on pain. Animal Sentience 2016.064.

Price, D.D., Verne, G.N. and Schwartz, J.M. (2006) Plasticity in brain processing and modulation of pain. Progr. Brain Res. 157: 333-352.

Riddoch, G. (1917) On the relative perceptions of movement and a stationary object in certain visual disturbances due to occipital injuries. Proc. Roy. Soc. Med. 10:13-34.

Safina, C. (2016) Fish pain: A painful topic. Animal Sentience 2016.066.

Walters, E.T. (2016) Pain-capable neural substrates may be widely available in the animal kingdom. Animal Sentience 2016.063.

Weiskrantz, L. (1996) Blindsight revisited. Curr. Opin. Neurobiol. 6:215-220.

Weiskrantz, L., Warrington, E.K., Sanders, M.D. and Marshall, J. (1974) Visual capacity in the hemianopic field following a restricted occipital ablation. Brain 97:709-728.

Weiss, N., Lawson, H.C., Greenspan, J.D., Ohara, S. and Lenz, F.A. (2005) Studies of the human ascending pain pathways. Thalamus \& Related Systems 3.01:71-86.

Willis, W.D. (1985) Nociceptive pathways: Anatomy and physiology of nociceptive ascending pathways. Phil. Trans. R. Soc. Lond. B 308:253-268.

Yager, D., Sharma, S.C., and Grover, B.G. (1977) Visual function in goldfish with unilateral and bilateral tectal ablation. Brain Res. 137:267-275. 